\title{
Prognostic significance of preoperative radiotherapy in stage II and III rectal cancer patients: A Strobe-compliant study of SEER 18 registries database (1988-2011)
}

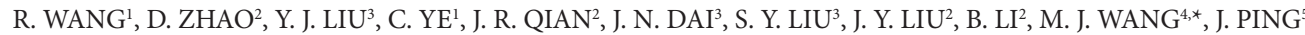

${ }^{1}$ Department of Gastroenterology, West China Hospital, Sichuan University, Chengdu, China; ${ }^{2}$ Department of General Surgery, Karamay Municipal Peoples' Hospital, Karamay, China; ${ }^{3}$ West China School of Medicine, Sichuan University, Chengdu, China; ${ }^{4}$ Department of Gastrointestinal Surgery, West China Hospital, Sichuan University, Chengdu, China; ${ }^{5}$ Division of Epidemiology, Vanderbilt University Medical Center, Nashville, TN, USA

${ }^{*}$ Correspondence: wangmojin2001@163.com

Received January 12, 2019 / Accepted April 16, 2019

\begin{abstract}
Preoperative radiation therapy has been regarded as the optional neoadjuvant treatment to decrease local recurrence of rectal cancer in addition to surgery. However, its benefit in survival remained obscure. This study was aimed to measure the efficacy of preoperative radiation therapy for survival in stage II and III rectal cancer patients. Retrospective cohort study used the database of Surveillance, Epidemiology and End Results program of the National Cancer Institute in the United States from 1988 to 2011. A total of 49,439 patients diagnosed with primary rectal cancer who underwent surgery were included. Clinicopathological characteristics and rectal cancer-specific survival between surgery alone group and surgery plus preoperative radiation therapy group were compared. Rectal cancer patients in surgery plus preoperative radiation therapy group had significantly better survival than those in surgery alone group $(72.70 \%$ vs. $66.61 \%$, p $<0.001)$, as well as stratified by stages (stage II: $77.4 \%$ vs. $74.3 \%$, p $<0.001$; stage III: $68.3 \%$ vs. $58.6 \%$, p $<0.001$ ). However, this beneficial impact was only observed after 2000s ( $<<0.001)$. Multivariate survival analysis revealed that preoperative radiation therapy was an independent predictor for better survival in stage III (hazard ratio, 0.795; 95\% CI, 0.753-0.840; $\mathrm{p}<0.001$ ), but not in stage II $(\mathrm{p}=0.70)$. Preoperative radiation therapy might bring a better survival in stage II and III rectal cancer patients, but only as an independent predictor for stage III patients. As time progressed, preoperative radiation therapy might yield more profit for stage II and III rectal cancer patients.
\end{abstract}

Key words: preoperative radiotherapy, rectal cancer, survival, Surveillance, Epidemiology and End Results database

Colorectal cancer remains the third most commonly diagnosed cancer and the second leading cause of cancer related death in the United States, leading to a huge medical investment as a health burden. Just in 2016, an estimated 49,190 patients died from colorectal cancer. During the same year, about 39,220 new cases of rectal cancer (RC) occurred in the United States (58.9\% were male) [1,2]. Different from colon cancer, the close proximity of the rectum to pelvic structures and organs, the absence of a serosa surrounding the lower rectum and the technical difficulties associated with obtaining sufficient surgical margins led to higher rate local recurrence of RC. Reports showed that $15-35 \%$ stage II and III patients would develop local recurrence after radical surgery. Even after the application of total mesorectal excision (TME), the local recurrence still remained as high as $5-10 \%$ [3]. Therefore, attempts to application of neoadju- vant therapy for stage II (T3-4, node-negative disease with tumor penetration through the muscle wall) or stage III (node-positive disease without distant metastasis) have been made to reduce local recurrence related death and improve prognosis of RC patients.

Preoperative radiation therapy (PRT) has been regarded as the revolutionary neoadjuvant treatment to decrease the rate of local recurrence of RC compared with surgery alone even after TME, and might be associated with the rapidly reducing perioperative mortality in recent years [4]. Thus PRT has been recommended in the NCCN guideline for locally advanced RC patients [1]. Nonetheless, the benefit of PRT in survival did not seem to meet the expectations [4], although the use of pelvic PRT in the treatment of patients with stage II/III RC continued to evolve. Addition of chemotherapy concurrent with PRT was demonstrated to bring possible benefit to local 
PRT sensitization, reduction of local recurrence and increase of pathologic complete response rate [5]. However, preoperative concurrent chemoradiotherapy not only did not benefit disease free or survival in resectable stage II and III RC as well, but also increased acute grade 3/4 treatment related toxicity [4, 6-10]. Inclusion of combined RC patients with stage II and III in various studies and the likely biases associated with single-institution experiences or limit cohort sizes might contributed to the disappointing results measuring survival of PRT. Whether PRT has the beneficial effect for survival in both stage II or III RC is worth exploration.

In this study, we analyzed the clinicopathological characteristics of stage II and III RC patients who underwent surgery and measured the actual efficacy of PRT in time course. Meanwhile, we established the prognostic factors related to the survival of stage II and III RC patients to test the possible efficacy of PRT.

\section{Patients and methods}

SEER database. All RC patients at stage II and III who underwent surgery from SEER 18 Registries database (1988 to 2011) were included. The SEER 18 Registries database collected patients' information of cancer incidence and survival representing approximately $30 \%$ of the United States population (http://seer.cancer.gov/about/overview.html). The information covered the records including demographics (age at diagnosis and gender), year of diagnosis, tumor characteristics (numbers, TNM stage, histological type, tumor grade) and treatments (surgery and receipt of PRT).

Table 1. Clinicopathological characteristics of rectal cancer patients.

\begin{tabular}{|c|c|c|c|}
\hline Characteristic & $S$ n $(\%)$ & S+PRT n (\%) & p-value \\
\hline Total number & $31796(64.3)$ & $17643(35.7)$ & \\
\hline \multicolumn{4}{|l|}{ Gender * } \\
\hline Male & $17079(53.7)$ & $11016(62.4)$ & \multirow{2}{*}{$<0.001$} \\
\hline Female & $14717(46.3)$ & $6627(37.6)$ & \\
\hline Age $($ years mean $\pm S D){ }^{\#}$ & $69.78 \pm 15.02$ & $60.35 \pm 12.74$ & $<0.001$ \\
\hline \multicolumn{4}{|l|}{ Tumor numbers * } \\
\hline Single & $21993(69.2)$ & $14401(81.6)$ & \multirow{2}{*}{$<0.001$} \\
\hline Multiple & $9803(30.8)$ & $3242(18.4)$ & \\
\hline \multicolumn{4}{|l|}{ TNM stage * } \\
\hline II & $16224(51.0)$ & $8523(48.3)$ & \multirow{2}{*}{$<0.001$} \\
\hline III & $15572(49.0)$ & $9120(51.7)$ & \\
\hline \multicolumn{4}{|l|}{ Histological Type * } \\
\hline Mucinous & $2620(8.4)$ & $1886(10.8)$ & \multirow{2}{*}{$<0.001$} \\
\hline Non-mucinous & $28737(91.6)$ & $15557(89.2)$ & \\
\hline Missing & 439 & 200 & \\
\hline \multicolumn{4}{|l|}{ Grade * } \\
\hline Well & $2097(6.8)$ & $1085(6.9)$ & \multirow{4}{*}{0.09} \\
\hline Moderately & $23190(75.6)$ & $11786(74.7)$ & \\
\hline Poorly+undifferentiated & $5418(17.6)$ & $2912(18.5)$ & \\
\hline Missing & 1091 & 1860 & \\
\hline
\end{tabular}

Note: ${ }^{\star}$ Chi-square test; ${ }^{\#}$ Independent t-test.
The version of SEER database used in this study was released in April 2014 (November 2013 submission).

Ethics statements. Data from SEER contained no identifiers and were publicly available. Permission was gained to access the research data files with the reference number 10058-Nov2013. Since it was a retrospective research and it required no interaction with human subjects or use of any personal identifying information, the informed consent was not needed. But the analysis was approved by the institutional review board of West China Hospital in China.

Patients selection. Patients with primary RC (C20.9) based on the International Classification of Diseases for Oncology (third edition, ICD-O-3) coding schema were included in this study. Data from eligible patients were stratified by whether they had PRT: S group (patients only received surgery) and S+PRT group (patients received PRT followed by surgery).

Data analysis. Tumor histological types were identified as mucinous cancer including signet-ring cell cancer (MC: 8480, 8481, 8490) and non-mucinous cancer (NMC: 8010, 8140-8141, 8144-8145, 8210-8211, 8220-8221, 8230-8231, 8260-8263). Histological grades were classified as well differentiated (G1), moderately differentiated (G2), poorly differentiated (G3), and undifferentiated ones (G4). TNM classification was restaged according to the criteria described in the American Joint Committee on Cancer (AJCC) Cancer Staging Manual, 7th edition, 2010. The 5-year rectal cancerspecific survival (RC-SS) rates and RC-SS time were measured from the time of diagnosis to the date of rectal cancer-specific death or the end of follow-up (cutoff date: December 2011). Patients were excluded if they had in situ or incomplete information. Clinicopathological characteristics and survival outcome were compared between S group and S+PRT group.

Statistical analysis. Independent t-test and Chi-square test were used to compare clinicopathological characteristics. Continuous data was exhibited as mean \pm standard deviation. Kaplan-Meier estimates were used to generate survival curves. Differences between the curves were analyzed using log-rank test. Multivariate Cox proportional hazard regression models were built and applied for the analysis of each variable on survival. The data was presented with $\mathrm{HR}$ and 95\% CI. All statistical tests were performed 2-sided. A p-value $<0.05$ was considered to be statistically significant. Statistical analyses were performed using R software system for statistical computing (version 3.1.2, http://www.r-project.org/).

\section{Results}

Demographic and clinical characteristics of patients with or without preoperative radiation therapy. A total of 49,439 eligible patients diagnosed with primary RC who underwent surgery from 1988 to 2011 in SEER database were analyzed. The basic characteristics of S group and S+PRT group are shown in Table 1 . Only $35.7 \%$ patients received PRT. Higher percentages of male $(\mathrm{p}<0.001)$ and patients with 
younger age $(\mathrm{p}<0.001)$ were in $\mathrm{S}+\mathrm{PRT}$ group compared with $S$ group. Patients with only single source of tumor $(p<0.001)$, diagnosed in stage III $(\mathrm{p}<0.001)$ and with mucinous $(\mathrm{p}<0.001)$ were more frequent in $\mathrm{S}+\mathrm{PRT}$ group than in $\mathrm{S}$ group.

Efficacy of preoperative radiation therapy in stage II and III patients. The better 5-year RC-SS rate was presented in S+PRT group compared with S group $(72.70 \%$ vs. $66.61 \%$, $\mathrm{p}<0.001$, Figure 1$)$. Further RC-SS analyses were stratified by each stage. After applied the PRT, patients with both stage II ( $77.4 \%$ vs. $74.3 \%, \mathrm{p}<0.001$, Figure 2$)$ and III $(68.3 \%$ vs. $58.6 \%, \mathrm{p}<0.001$, Figure 3 ) exhibited a more desirable 5 -year RC-SS than those who underwent only surgery.

Efficacy of preoperative radiation therapy in Time Course. Due to the low frequency, PRT has been rarely studied in time course. Since 2000s a wide variety of improvements with respect of diagnosis and treatments etc. in RC has happened, therefore the RC-SS was tested accordingly. We divided the data into two time phases: 1) from 1988 to 1999; 2) from 2000 to 2011, based on the time point (since 2000s) for the widely spread of revolutionary operational strategy change according to TME. After 2000s, the RC-SS was significantly better when compared with the period of 1988-1999 ( $<<0.001$, Supplementary Figure S1A). Whether in patients with only surgery or in patients with combination of surgery and PRT, patients treated after 2000s at both, stage II and III, were presented with preferable survival outcome $(\mathrm{p}<0.001$, Supplementary Figure S1B and $1 \mathrm{C})$. The time course (1988-1999 vs. 2000-2011) was also an independent prognostic factor for survival of RC at either stage II or III $(\mathrm{p}<0.001$, Table 2$)$.

Table 2. Multivariate survival analysis of prognostic factors in stage II and III rectal cancer patients.

\begin{tabular}{lcc}
\hline Variable & HR (95\%CI) & p-value \\
\hline II stage & & \\
$\quad$ Surgery+ PRT (yes vs. no) & $0.987(0.925-1.054)$ & 0.70 \\
Gender (male vs. female) & $1.033(0.974-1.096)$ & 0.28 \\
Age ( $\geq 70$ vs $<$ 70) & $1.864(1.754-1.980)$ & $<0.001$ \\
Tumor numbers (multiple vs. single) & $0.659(0.604-0.720)$ & $<0.001$ \\
Histological type (MC vs. NMC) & $1.384(1.254-1.528)$ & $<0.001$ \\
Grade (poorly+ undifferentiated & $1.339(1.237-1.450)$ & $<0.001$ \\
vs. well + moderately) & & \\
Time course (1988-1999 vs. 2000-2011) & $1.314(1.105-1.501)$ & $<0.001$ \\
III stage & & \\
Surgery+ PRT (yes vs. no) & $0.795(0.753-0.840)$ & $<0.001$ \\
Gender (male vs. female) & $1.098(1.045-1.153)$ & $<0.001$ \\
Age ( $\geq 70$ vs $<70)$ & $1.797(1.710-1.889)$ & $<0.001$ \\
Tumor numbers (multiple vs. single) & $0.641(0.591-0.695)$ & $<0.001$ \\
Histological type (MC vs. NMC) & $1.427(1.326-1.537)$ & $<0.001$ \\
Grade (poorly + undifferentiated & $1.548(1.467-1.634)$ & $<0.001$ \\
vs. well + moderately) & $1.153(1.087-1.325)$ & $<0.001$ \\
\hline Time course (1988-1999 vs. 2000-2011) & &
\end{tabular}

Note: $\mathrm{PRT}=$ preoperative radiotherapy; $\mathrm{MC}=$ mucinous cancer; $\mathrm{NMC}=$ non-mucinous cancer.

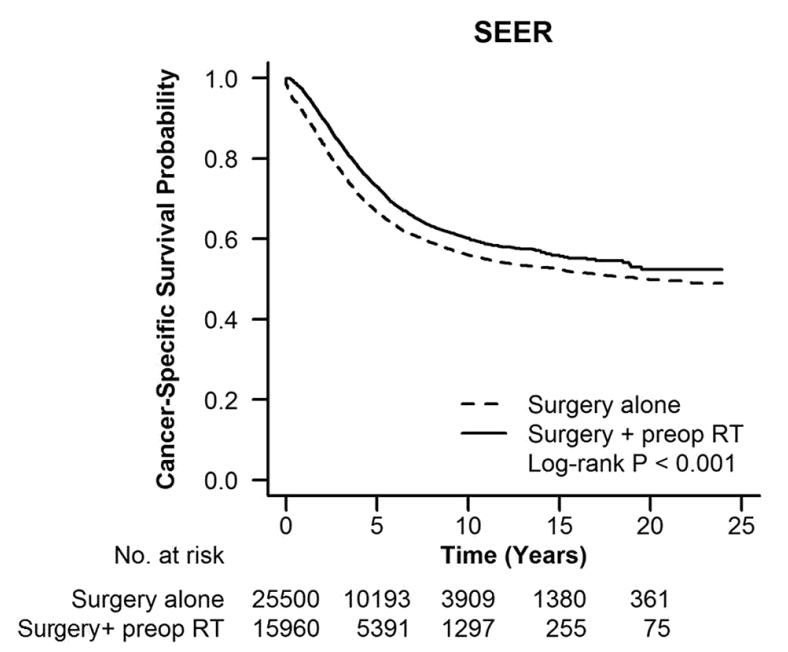

Figure 1. Comparison of RC-SS of RC patients between S and S+PRT group.

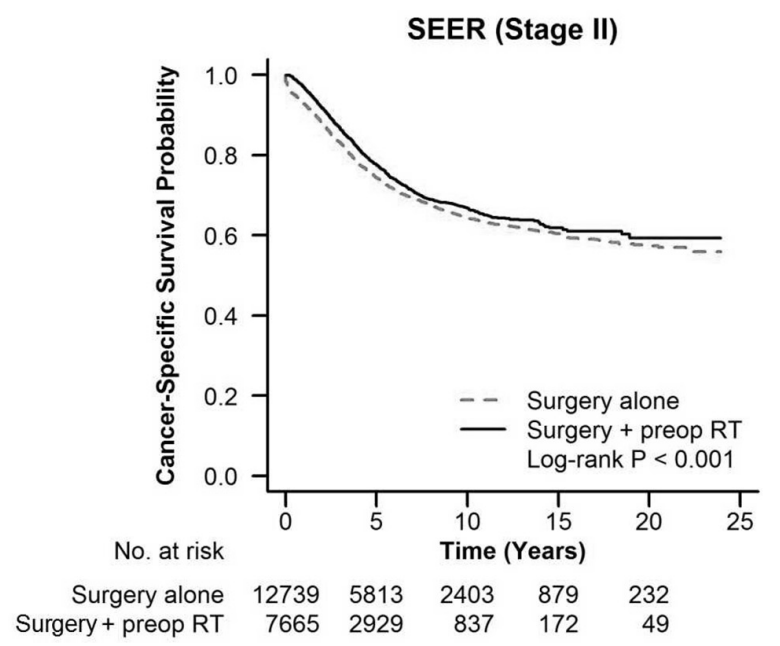

Figure 2. Comparison of RC-SS in stage II RC patients between $S$ and S+PRT group.

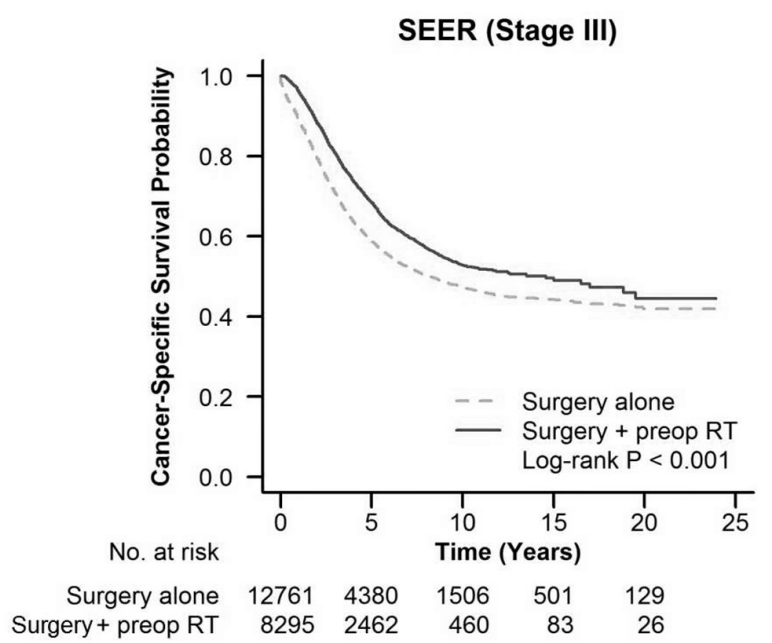

Figure 3. Comparison of RC-SS in stage III RC patients between $S$ and S+PRT group. 
Further analysis into the time course was quite intriguing. In the period of 1988-1999, PRT did not showed significant advantage over but otherwise inferior to therapy with only surgery $(\mathrm{p}=0.02$, Supplementary Figure S2A). The inferiority of PRT was more obvious in patients with stage II ( $<<0.001$, Supplementary Figure S2B). On the contrary, since 2000s, PRT demonstrated a significant benefit in survival of RC either in stage II or III ( $<<0.001$, Supplementary Figure S3).

Prognostic implications of preoperative radiation therapy in stage II and III rectal cancer patients. Multivariate survival analysis of prognostic factors for RC patients at stage II and III using Cox model is shown in Table 2. From this analysis, age $(\geq 70)$, histological type of mucinous cancer, poorly differentiation and single source of tumor worsened the survival in both stage II and III RC patients $(\mathrm{p}<0.001)$. However, PRT and gender of female as the independent predictors for better prognosis were only observed in stage III patients other than stage II. It suggested that stage III patients benefited from PRT [hazard ratio (HR), 0.795; 95\% confidence interval (CI), 0.753-0.840; $\mathrm{p}<0.001]$, while PRT in stage II patients remained consideration $(\mathrm{p}=0.70)$.

\section{Discussion}

At the end of the 1980s and during the 1990s, evidences were growing that an addition of PRT to exclusive surgical treatment of RC could improve patients' prognosis, therefore, as a mainstay of treatment alongside surgery. In consideration of reducing local recurrences for the majority of patients with stage II or stage III RC, PRT was recommended in the guidelines in addition to curative intent surgery. But it was also found associated with increased toxicity (e.g. radiation-induced injury, hematologic toxicities) and barely satisfactory benefit for survival in partial RC patients $[4,11,12]$. In view of this situation, the prognostic implications of PRT in stage II and stage III RC patients needed deeper exploration. To avoid the biases associated with single-institution experiences or limit sample sizes, in this population-based study we analyzed 49,439 RC patients with stage II and III from the national data of SEER program between 1988 and 2011. Only $35.7 \%$ patients were subjected to PRT. This result was similar to a previous literature which identified 35.5\% patients treated with PRT from 1998 to 2007 in the SEER tumor registry identifying 22,136 stage II/III RC patients undergoing surgery [13]. Although a significant increase in the use of PRT occurred from $17 \%$ in 1998, PRT for stage II and III RC patients was still not delivered on a routine basis.

Among our analyzed population, patients with characteristics including male, stage III, and mucinous carcinoma were more frequent to undertake PRT in accordance with previous researches [13]. This was probably because those features were well-recognized undesirable factors for higher recurrence and metastasis in RC leading to a worse survival, therefore, needed more aggressive preoperative neoadjuvant therapy $[14,15]$. Meanwhile, reports showed that patients with younger age might present with more tumors located in rectum, fewer cases with multiple tumors, later stage, more mucinous carcinoma and lymph nodes examined [14, 16]. With those clinical features as well as less comorbidities, higher extensive lymphadenectomy rates, lower risk of postoperative complications and better toleration of PRT related toxicity, doctors might prefer to apply comprehensive treatment including PRT to young RC patients. With barely none reports previously, our research revealed that less than one-third $(24.85 \%, 3,242 / 13,054)$ of patients with multiple tumors were subjected to PRT. The reason remained obscure. This was probably because higher incidences of second primary cancers might develop after PRT, let alone those patients with original multiple tumors in the beginning [17], as well as more acute and late side effects of PRT for multiple tumors. Besides the undisputed importance of acute toxicity, long-term follow-up data from the Dutch trial [12] or Swedish cohorts [18] exhibited a higher rate of fecal and urinary incontinence even after short course radiotherapy compared to surgery alone, thus impairing quality of life. The distribution of patients into PRT group in this research might be a display of the goal to optimize disease-free survival while minimizing the risk of local recurrence and toxicity with the PRT theoretically.

Nevertheless, the efficacy of PRT in RC survival was not yet certain. Reports have confirmed that PRT was superior over surgery alone in local control. A meta-analysis containing nineteen trials comparing PRT versus surgery alone for the management of localized rectal carcinoma showed that optimal PRT could improve local recurrences [19]. Sensitivity analyses suggested greater benefits in patients treated with biologic effective dose (BED) $>30$ Gy (10) than a BED of $\leq 30$ Gy (10) and multiple field RT techniques, independent of the schedule of fractionation used [20]. However, this meta-analysis did not mention its impact on survival. Several European studies have investigated the efficacy of a shorter course PRT (25 Gy over 5 days), not combined with chemotherapy, for the treatment of RC. A 12-year follow-up of multicenter, randomized controlled TME trial in Dutch revealed that preoperative short-term radiotherapy reduced 10-year local recurrence by more than $50 \%$ relative to surgery alone without a survival benefit [21]. Similar results were presented in a number of other studies which inquired into the effectiveness of short-course PRT in patients with RC staged T1-3 and demonstrated that survival was not significantly affected despite improvements in local control of disease [22-24]. On the contrary, in a Swedish RC trial, the five-year survival rate was $58 \%$ in the radiotherapy-plus-surgery group and $48 \%$ in the surgery-alone group $(\mathrm{p}=0.004)$ [25]. The confusing results of survival might be attributed to relatively small sample size, inclusion of diverse TMN stage, etc. To address this problem, our study was by far the first analysis including only stage II and III eligible RC patients and largest cohort size to date. Therefore, it might offer a relatively more convincing evidence. Our study exhibited that the 5 year RC-SS was more 
desirable after applying PRT than surgery alone in stage II and III patients, reinforcing the necessary for application of PRT in guidelines, especially in high risk population.

Interestingly, in further multivariate survival analysis of prognostic factors stratified by tumor stages, which were rarely reported in previous studies, we found that except for old age, histological type of mucinous cancer, poor differentiation and single source of tumor served as independent predictors for worse survival in both stage II and III RC patients, in accordance with other analyses from SEER (19882011) and SEER (1973-2011) $[15,26]$. PRT as an independent predictor for better prognosis was only observed in stage III other than stage II, which was rarely reported before. It suggested that PRT might benefit prominently in stage III RC patients. The advantage of PRT in stage III was also seen in the TME trial. For stage III RC patients with a negative circumferential resection margin, 10-year survival was 50\% in the short-term PRT group versus $40 \%$ in the surgery-alone group ( $p=0.03$ ) [21]. The reason for this distinct of PRT in stage II and III was unknown. A combined analysis of SEER and data from Fudan University Shanghai Cancer Center indicated that increased number of negative lymph nodes (NLN) was associated with improved cancer specific survival in pathological IIIB and IIIC RC treated with PRT [27, 28], suggesting that PRT might significantly improve NLN status for a better survival of less distance lymph node metastasis in stage III RC patients $[29,30]$.

On the other hand, the relative inferiority of PRT in stage II RC patients was also discovered in another multivariate analysis from SEER database (1998-2008). It showed that PRT was not significantly associated with improved cancer survival in T3N0 (IIA) RC patients (HR=0.863; 95\% CI, $0.715-1.043 ; \mathrm{p}=0.13$ ) [31]. Some researchers thought that these stage II RC patients with no lymph node invasion and lower risk of local recurrence (clear margins and favorable prognosis features) might be adequately treated with surgery and adjuvant chemotherapy. But many patients were understaged by preoperative clinical imaging and subsequently proved to have positive lymph nodes in the surgical specimens. PRT was still considered valuable. Worth mentioning was that PRT impact was not always suboptimal. After 2000s, PRT demonstrated a significant benefit in the survival of stage II RC when compared with data from 1988-1999. The credit for this improvement might go to the widespread, rapid adoption of PRT for locally advanced RC and related progress in radiation dosage, schedule, interval to surgery and combined with or without chemotherapy [32].

Because the AJCC stage was not available in the database until 1988, we did not include RC patients between 1973 and 1988 into this study. Although SEER data was considered as a cancer registry data meeting international standard with good quality and completeness, a few important limitations of the current study deserve a notice. Firstly, SEER registry did not record the detailed information concerning the dose or duration of chemotherapy, but also the actual dose or cycles of radiation received by each patient. Therefore, we were unable to take differences of PRT and chemotherapy practice into account over the study period. Secondly, in clinical scenario of emergency, certain patients with bowel obstruction, intestinal perforation, ischemic necrosis or severe infection etc., might undergo an emergency surgery and miss the precious opportunity of PRT, thereby comprising a large share in solo-surgery group. Furthermore, the data did not contain several records like lymphatic/vascular invasion, quality of surgery and local/distant recurrence status. Unfortunately, these prognostic factors of stage II and III RC patients could not be analyzed in our study. Although cancer recurrences were not available in present study, RC-SS was a reasonable surrogate of RC-specific outcome. Despite these, the results of the current research might provide some information for future studies of PRT in stage II and III RC patients. In order to obtain a more definitive conclusion, further larger multicenter randomized controlled trial should be carried out.

Taken together, the evidences from this study reflected that the implement rate of PRT was only $35.7 \%$ in stage II and III RC patients from database of SEER during 1988-2011. More patients with the clinicopathological characteristics of male, younger age, single source of tumor, stage III and mucinous rectal cancer undertook PRT. PRT might bring a better survival in stage II and III RC patients, but only as an independent predictor for stage III patients. As time progressed, PRT might yield more profit for stage II RC patients. But further studies are still needed to provide additional insight into the selected criterion instead of generalized indications for RC patients at stage II and III to receive optimized PRT.

Supplementary information is available in the online version of the paper.

Acknowledgements: This work was supported by the grants from the National Scientific Foundation of China (Grant NO. 81401949, 8130035) and Key Research projects of Department of science and Technology of Sichuan Province, China (Grant no.2018SZ0403). Popularization and Application Project of Health and Family Planning Commission of Sichuan Province, China (Grant no.18PJ387).

\section{References}

[1] NCCN. National Comprehensive Cancer Network. NCCN Clinical Practice Guidelines in Oncology. Rectal Cancer. Version 3. 2017. https://www.nccn.org/professionals/physician_gls/f_guidelines.asp

[2] SIEGEL RL, MILLER KD, JEMAL A. Cancer statistics, 2016. CA Cancer J Clin 2016; 66: 7-30. https://doi.org/10.3322/ caac. 21332

[3] GLIMELIUS B, TIRET E, CERVANTES A, ARNOLD D. Rectal cancer: ESMO Clinical Practice Guidelines for diagnosis, treatment and follow-up. Ann Oncol 2013; 24: vi81vi88. https://doi.org/10.1093/annonc/mdt240 
[4] RAHBARI NN, ELBERS H, ASKOXYLAKIS V, MOTSCHALL E, BORK U et al. Neoadjuvant radiotherapy for rectal cancer: meta-analysis of randomized controlled trials. Ann Surg Oncol 2013; 20: 4169-4182. https://doi. org/10.1245/s10434-013-3198-9

[5] UEHARA K, NAGINO M. Neoadjuvant treatment for locally advanced rectal cancer: a systematic review. Surg Today 2016; 46: 161-168. https://doi.org/10.1007/s00595-0151218-z

[6] GERARD JP, CONROY T, BONNETAIN F, BOUCHE O, CHAPET O et al. Preoperative radiotherapy with or without concurrent fluorouracil and leucovorin in T3-4 rectal cancers: results of FFCD 9203. J Clin Oncol 2006; 24: 46204625. https://doi.org/10.1200/JCO.2006.06.7629

[7] BOSSET JF, COLLETTE L, CALAIS G, MINEUR L, MAINGON P et al. Chemotherapy with preoperative radiotherapy in rectal cancer. N Engl J Med 2006; 355: 1114-1123. https:// doi.org/10.1056/NEJMoa060829

[8] CEELEN WP, VAN NIEUWENHOVE Y, FIERENS K. Preoperative chemoradiation versus radiation alone for stage II and III resectable rectal cancer. Cochrane Database Syst Rev 2009; 1: CD006041. https://doi.org/10.1002/14651858. CD006041.pub2

[9] MCCARTHY K, PEARSON K, FULTON R, HEWITT J. Pre-operative chemoradiation for non-metastatic locally advanced rectal cancer. Cochrane Database Syst Rev 2012; 12: CD008368. https://doi.org/10.1002/14651858.CD008368. pub2

[10] DE CALUWE L, VAN NIEUWENHOVE Y, CEELEN WP. Preoperative chemoradiation versus radiation alone for stage II and III resectable rectal cancer. Cochrane Database Syst Rev 2013; 2: CD006041. https://doi.org/10.1002/14651858. CD006041.pub3

[11] LAI LL, FULLER CD, KACHNIC LA, THOMAS CR JR. Can pelvic radiotherapy be omitted in select patients with rectal cancer? Semin Oncol 2006; 33: S70-74.

[12] PEETERS KC, VAN DE VELDE CJ, LEER JW, MARTIJN $\mathrm{H}$, JUNGGEBURT JM et al. Late side effects of short-course preoperative radiotherapy combined with total mesorectal excision for rectal cancer: increased bowel dysfunction in irradiated patients--a Dutch colorectal cancer group study. J Clin Oncol 2005; 23: 6199-6206. https://doi.org/10.1200/ JCO.2005.14.779

[13] FITZGERALD TL, BISWAS T, O’BRIEN K, ZERVOS EE, WONG JH. Neoadjuvant radiotherapy for rectal cancer: adherence to evidence-based guidelines in clinical practice. World J Surg 2013; 37: 639-645. https://doi.org/10.1007/ s00268-012-1862-z

[14] WANG R, WANG MJ, PING J. Clinicopathological Features and Survival Outcomes of Colorectal Cancer in Young Versus Elderly: A Population-Based Cohort Study of SEER 9 Registries Data (1988-2011). Medicine (Baltimore) 2015; 94: e1402. https://doi.org/10.1097/MD.0000000000001402

[15] WANG MJ, PING J, LI Y, HOLMQVIST A, ADELL G et al. Prognostic Significance and Molecular Features of Colorectal Mucinous Adenocarcinomas: A Strobe-Compliant Study. Medicine (Baltimore) 2015; 94: e2350. https://doi. org/10.1097/MD.0000000000002350
[16] WANG MJ, PING J, LI Y, ADELL G, ARBMAN G et al. The prognostic factors and multiple biomarkers in young patients with colorectal cancer. Sci Rep 2015; 5: 10645. https:// doi.org/10.1038/srep10645

[17] SMITH-GAGEN J, GOODWIN GA 3RD, TAY J. Multiple primary tumors following stage II and III rectal cancer in patients receiving radiotherapy, 1998-2010. J Cancer Res Clin Oncol 2014; 140: 949-955. https://doi.org/10.1007/s00432$014-1647-\mathrm{x}$

[18] POLLACK J, HOLM T, CEDERMARK B, ALTMAN D, HOLMSTROM B et al. Late adverse effects of short-course preoperative radiotherapy in rectal cancer. Br J Surg 2006; 93: 1519-1525. https://doi.org/10.1002/bjs.5525

[19] WONG RK, TANDAN V, DE SILVA S, FIGUEREDO A. Preoperative radiotherapy and curative surgery for the management of localized rectal carcinoma. Cochrane Database Syst Rev 2007; 2: CD002102. https://doi.org/10.1002/14651858. CD002102.pub2

[20] VIANI GA, STEFANO EJ, SOARES FV, AFONSO SL. Evaluation of biologic effective dose and schedule of fractionation for preoperative radiotherapy for rectal cancer: meta-analyses and meta-regression. Int J Radiat Oncol Biol Phys 2011; 80: 985-991. https://doi.org/10.1016/j. ijrobp.2010.03.008

[21] VAN GIJN W, MARIJNEN CA, NAGTEGAAL ID, KRANENBARG EM, PUTTER $\mathrm{H}$ et al. Preoperative radiotherapy combined with total mesorectal excision for resectable rectal cancer: 12-year follow-up of the multicentre, randomised controlled TME trial. Lancet Oncol 2011; 12: 575-582. https://doi.org/10.1016/S1470-2045(11)70097-3

[22] KAPITEIJN E, MARIJNEN CA, NAGTEGAAL ID, PUTTER H, STEUP WH et al. Preoperative radiotherapy combined with total mesorectal excision for resectable rectal cancer. N Engl J Med 2001; 345: 638-646. https://doi. org/10.1056/NEJMoa010580

[23] PEETERS KC, MARIJNEN CA, NAGTEGAAL ID, KRANENBARG EK, PUTTER $\mathrm{H}$ et al. The TME trial after a median follow-up of 6 years: increased local control but no survival benefit in irradiated patients with resectable rectal carcinoma. Ann Surg 2007; 246: 693-701. https://doi. org/10.1097/01.sla.0000257358.56863.ce

[24] SEBAG-MONTEFIORE D, STEPHENS RJ, STEELE R, MONSON J, GRIEVE R et al. Preoperative radiotherapy versus selective postoperative chemoradiotherapy in patients with rectal cancer (MRC CR07 and NCIC-CTG C016): a multicentre, randomised trial. Lancet 2009; 373: 811-820. https://doi.org/10.1016/S0140-6736(09)60484-0

[25] SWEDISH RECTAL CANCER TRIAL, CEDERMARK B, DAHLBERG M, GLIMELIUS B, PAHLMAN L et al. Improved survival with preoperative radiotherapy in resectable rectal cancer. N Engl J Med 1997; 336: 980-987. https://doi. org/10.1056/NEJM199704033361402

[26] LING CR, WANG R, WANG MJ, PING J, ZHUANG W. Prognosis and value of preoperative radiotherapy in locally advanced rectal signet-ring cell carcinoma. Sci Rep 2017; 7: 45334. https://doi.org/10.1038/srep45334 
[27] LI Q, ZHUO C, CAI G, LI D, LIANG L et al. Increased number of negative lymph nodes is associated with improved cancer specific survival in pathological IIIB and IIIC rectal cancer treated with preoperative radiotherapy. Oncotarget 2014; 5: 12459-12471. https://doi.org/10.18632/oncotarget. 2560

[28] LI X, LU H, XU K, WANG H, LIANG X et al. Negative lymph node count is an independent prognostic factor for patients with rectal cancer who received preoperative radiotherapy. BMC Cancer. 2017; 17: 227. https://doi.org/10.1186/ s12885-017-3222-8

[29] CHANG GJ, RODRIGUEZ-BIGAS MA, ENG C, SKIBBER JM. Lymph node status after neoadjuvant radiotherapy for rectal cancer is a biologic predictor of outcome. Cancer 2009; 115: 5432-5440. https://doi.org/10.1002/cncr.24622
[30] Li Q, Zhuo C, Liang L, Zheng H, Li D et al. Lymph node count after preoperative radiotherapy is an independently prognostic factor for pathologically lymph node-negative patients with rectal cancer. Medicine (Baltimore) 2015; 94: e395. https://doi.org/10.1097/MD.0000000000000395

[31] PENG LC, MILSOM J, GARRETT K, NANDAKUMAR G2, COPLOWITZ S et al. Surveillance, epidemiology, and end results-based analysis of the impact of preoperative or postoperative radiotherapy on survival outcomes for T3N0 rectal cancer. Cancer Epidemiol 2014; 38: 73-78. https://doi. org/10.1016/j.canep.2013.12.008

[32] MAK RH, MCCARTHY EP, DAS P, HONG TS, MAMON $\mathrm{HJ}$ et al. Adoption of preoperative radiation therapy for rectal cancer from 2000 to 2006: a Surveillance, Epidemiology, and End Results Patterns-of-Care Study. Int J Radiat Oncol Biol Phys 2011; 80: 978-984. https://doi.org/10.1016/j. ijrobp.2010.03.056 